\title{
Dental abnormalities in a patient with autosomal dominant hyper-IgE syndrome: A case control study
}

Marziyeh Heidarzadeh ${ }^{1}$, Atena Ramezanali Yakhchali ${ }^{2}$, Mohammad Gharagozlou ${ }^{3}$, Sepideh Darougar $^{4}$, Zahra Chavoshzadeh ${ }^{2}$, Mahnaz Jamee ${ }^{2}$, and Hossein Motedayyen ${ }^{1}$

${ }^{1}$ Kashan University of Medical Sciences

${ }^{2}$ Shahid Beheshti University of Medical Sciences

${ }^{3}$ Tehran University of Medical Sciences

${ }^{4}$ Islamic Azad University Tehran Medical Sciences

May 27, 2021

\begin{abstract}
Autosomal-dominant hyper-IgE (AD-HIES) is mainly characterized by eczematous dermatitis, staphylococcal skin abscesses, connective tissue defects, and elevated serum IgE. This disorder is largely associated with heterozygous dominant-negative mutations in STAT3 gene. Herein, we reported a patient with AD-HIES suffering from dental abnormality and allergic reactions.
\end{abstract}

Dental abnormalities in a patient with autosomal dominant hyper-IgE syndrome: A case control study

Authors

Marzieh Heidarzadeh Arani ${ }^{1}$, Atena Ramezanali Yakhchali $^{2}$, Mohammad Gharagozlou, MD $^{3}$, Sepideh Darougar, $\mathrm{MD}^{4}$, Zahra Chavoshzadeh, $\mathrm{MD}^{5}$, Mahnaz Jamee ${ }^{6,}{ }^{*}$, Hossein Motedayyen ${ }^{7}$ *

\section{Affiliations}

1. Department of Pediatrics, Kashan University of Medical Sciences, Kashan, Iran.

2. Shahid Beheshti University of Medical Sciences and Health Services, Tehran, Iran.

3. Department of Allergy and Clinical Immunology, Children's Medical Center, Tehran University of Medical Sciences, Tehran, Iran.

4. Department of Pediatrics, Tehran Medical Sciences Branch, Islamic Azad University, Tehran, Iran.

5. Immunology and Allergy Department, Mofid Children's Hospital, Shahid Beheshti University of Medical Sciences, Tehran, Iran.

${ }^{6}$. Pediatric Nephrology Research Center, Research Institute for Children's Health, Shahid Beheshti University of Medical Sciences, Tehran, Iran.

7. Autoimmune Diseases Research Center, Kashan University of Medical Sciences, Kashan, Iran.

${ }^{*}$ Corresponding Authors : Dr. Hossein Motedayyen, Autoimmune Diseases Research Center, Shahid Beheshti Hospital, Kashan University of Medical Sciences, 5th Kilometer of Ravand Road, Kashan, Iran, Fax: 00983155578011, Post Code: 8715973474, e-mail: hmotedayyen@gmail.com. Miss. Mahnaz Jamee, Pediatric Nephrology Research Center, Research Institute for Children's Health, Shahid Beheshti University 
of Medical Sciences, Arabi Ave, Daneshjoo Blvd, Velenjak, Tehran, Iran. Post Code: 19839-63113, e-mail: mahnaz.jamee@gmail.com.

Running title: Dental implications in AD-HIES.

\section{Key Clinical Message:}

Dental abnormalities and other clinical complications of HIES are largely associated with heterozygous dominant-negative mutations inSTAT3 . Early diagnosis and management of these complications can improve life quality of patients.

Keywords: Hyper-IgE syndrome, STAT3, Recurrent infections, Dental abnormality, Allergic reactions.

\section{Introduction}

Autosomal dominant hyper-IgE syndrome (AD-HIES), also known as Buckley or Job's syndrome, is a rare multisystem disorder with both immunologic and nonimmunological characteristics. ${ }^{1}$ This disorder is largely associated with heterozygous dominant-negative mutations in signal transducer and activator of transcription 3 (STAT3 ) gene and characterized by eczematoid rashes, staphylococcal skin abscesses, connective tissue defects, and extremely elevated IgE serum level. ${ }^{2-5}$

Abnormalities in the dentition are often observed in patients with AD-HIES, such as retention of primary teeth together with ectopic eruption or non-eruption of permanent teeth. ${ }^{6}$ In addition to dental abnormalities, some oral cavity defects have been reported for AD-HIES, including deep grooves on the tongue, buccal mucosa with multiple fissures, central ridges and fissures of the palate and a high arched palate. ${ }^{7}$

In this report, we reported dental abnormalities observed in an AD-HIES patient suffering from dermatitis, asthma, eczema, anaphylaxis, and refractory sinusitis.

\section{Case history}

The case was a 14-year-old boy referred to the allergy and immunodeficiency clinic of Shahid Beheshti hospital, Kashan, Iran. He suffered from acute asthma attacks and eczema. He was the third child of nonrelative parents. His problems began at the age of ten days when he experienced acute erythematous papules associated with excoriations and vesicles over the skin of glans following circumcision procedure. Facial dermatitis appeared and continued to involve other parts of his body at six months of age. A skin prick test was performed which was indicative of sensitivity reactions to milk, egg, and wheat. Therefore, an elimination diet including milk, egg, and wheat was recommended and started. At the age of 18 months, he had two episodes of cough, dyspnea, and wheezing, resembling asthma attacks which were followed by more exacerbations at older ages. These exacerbations were effectively controlled by inhaled corticosteroids. At the age of 12 years, the patient experienced an episode of anaphylaxis. He also suffered from recurrent sinusitis without pulmonary infections. Growth and development of patients were normal. However, primary teeth were retained which was accompanied by ectopic eruption of permanent teeth (Figure 1). Based on these findings, an underlying immunodeficiency disorder was considered. Some laboratory tests were carried out for investigating immunological situations of our case (Table. 1). The results of these tests revealed the serum level of $\mathrm{IgE}$ was significantly elevated $(1320 \mathrm{IU} / \mathrm{ml})$ which in association with his previous history proposed the possibility of hyper IgE syndrome (HIES). The patient was genetically studied to determine a possible defect in the STAT3 gene. The genetic analysis indicated that he had a heterozygous mutation in STAT3 with a nucleotide exchange of $1909 \mathrm{G}>\mathrm{A}$ in the exon 21 of STAT3 gene, leading to an amino acid change (V637M), previously known as the cause of the autosomal dominant type of hyper IgE syndrome (HIES).

Regarding the fact that our patient did not cooperate for further evaluation and only suffered from asthma, his treatment was continued by Symbicort $160 / 4.5 \mathrm{mg}$ /inhalation one puff twice daily. Our case is presently alive and in acceptable health condition.

\section{Discussion}


STAT3 is a critical regulator of multiple processes, including cellular proliferation, survival, differentiation, fetal development, cancer, wound healing, angiogenesis, autoimmunity and inflammation. ${ }^{5}$ Defect in its function results in multisystem disorders such as dental abnormalities and connective tissue defects. ${ }^{5}$ In the present study, we investigated a boy with acute asthma attacks, eczema, and dental abnormalities.

Abnormalities in the dentition are frequently observed in AD-HIES, although its mechanism(s) is not well identified yet. It is reported that STAT3 as a transcription factor play indispensable roles in signaling pathways of various cytokine such as IL-6, IL-10, IL-21, IL-22, and IL-23. ${ }^{8,9}$ Among these cytokines, IL-6 has a positive role in bone resorption through inducing osteoclastogenesis. ${ }^{10,}{ }^{11}$ Thus, impaired STAT3 signaling may disrupt the role of IL-6 in osteoporosis and thereby contribute to the retention of primary teeth in AD-HIES. ${ }^{12}$ In line with this notions, some reports have revealed that retention of deciduous teeth is owing to reduced resorption of tooth roots leading to failure of eruption of permanent teeth. ${ }^{7}$ Grimbacher et al evaluated 30 patients with HIES and 70 of their relatives and reported primary teeth retention in $72 \%$ of them. ${ }^{13}$ In another study by O'Connel et al on 34 HIES patients, it was indicated that $75 \%$ of subjects older than 7 years had problem with permanent tooth eruption, in the form of prolonged retention of primary teeth or the need for extraction of primary teeth. ${ }^{14}$ In another report of 60 patients with the HIES, $76.7 \%$ of subjects suffered from intraoral lesions such as fibrotic bridges in the hard palate or keratotic plaques which were more prevalent than the characteristic facial traits. ${ }^{15}$ Considering the above findings in the literature, abnormal dentition in our patient is a common feature but as the prominent finding in an adolescent is particularly noteworthy.

Asthma and anaphylaxis were two atypical presentations in this patient. According to the literature, allergic manifestations including allergic rhinitis, asthma, urticaria, and anaphylaxis are not common clinical features in these patients despite their high serum IgE concentrations. Wjst et al tested whether STAT3 mutations were responsible for increased IgE levels in asthmatic children and found it unlikely. ${ }^{16}$ Furthermore, some studies have reported no significant association between STAT3 gene expression and either mild or severe refractory asthma. ${ }^{17}$ However, positive findings regarding an allergic background in our case including neonatal dermatitis with positive skin prick test to milk, egg, and wheat and a favorable response to an elimination diet, recurrent asthma exacerbations, as well as a history of anaphylaxis may denote an underlying atopic predisposition.

Taken together, our findings indicate that clinical evaluations of some atypical presentations, such as asthma and anaphylaxis, along with immunological and genetic analyses can be helpful to better diagnosis and management of AD-HIES, which can improve life quality of patients. Therefore, patients suffering from allergic and infectious diseases, autoimmune disorders, and dental abnormalities should be investigated by clinical, immunological, and genetic tests to determine possible mutations in STAT3 gene.

\section{Acknowledgments:}

The authors would like to give special thanks to Professor Bodo Grimbacher for performing the genetic study.

\section{Conflict of interest}

The authors declare that they have no competing interests.

\section{Author contribution}

Marzieh Heidarzadeh Arani and Atena Ramezanali Yakhchali contributed to the conceptualization, data curation, supervision, and writing the original draft; Mohammad Gharagozlou, Sepideh Darougar, Zahra Chavoshzadeh, and Mahnaz Jamee and Hossein Motedayyen reviewed and edited the final manuscript. All authors read and approved the final manuscript.

\section{Ethical approval}

Written informed consent was obtained from parents for the clinical data and photos of their child to be published. This study is approved by the Ethics Committee of Kashan University of Medical Sciences. 


\section{Data availability statement}

All data generated or analyzed during this study are included in this published case report.

\section{References}

1. Vinh DC, Sugui JA, Hsu AP, Freeman AF, Holland SM. Invasive fungal disease in autosomal-dominant hyper-IgE syndrome. The Journal of allergy and clinical immunology. 2010;125(6):1389.

2. Schimke LF, Sawalle-Belohradsky J, Roesler J, Wollenberg A, Rack A, Borte M, et al. Diagnostic approach to the hyper-IgE syndromes: immunologic and clinical key findings to differentiate hyper-IgE syndromes from atopic dermatitis. Journal of allergy and clinical immunology. 2010;126(3):611-7. e1.

3. Cruz-Portelles A, Estopiñan-Zuniga D. A new case of Job's syndrome at the clinic: A diagnostic challenge. Revista Portuguesa de Pneumologia (English Edition). 2014;20(2):107-10.

4. Fabre A, Marchal S, Barlogis V, Mari B, Barbry P, Rohrlich PS, et al. Clinical Aspects of STAT3 Gain-ofFunction Germline Mutations: A Systematic Review. J Allergy Clin Immunol Pract. 2019;7(6):1958-69.e9.

5. Fabre A, Marchal S, Barlogis V, Mari B, Barbry P, Rohrlich P-S, et al. Clinical aspects of STAT3 gain-of-function germline mutations: a systematic review. The Journal of Allergy and Clinical Immunology: In Practice. 2019;7(6):1958-69. e9.

6. Esposito L, Poletti L, Maspero C, Porro A, Pietrogrande MC, Pavesi P, et al. Hyper-IgE syndrome: dental implications. Oral surgery, oral medicine, oral pathology and oral radiology. 2012;114(2):147-53.

7. Yong PF, Freeman AF, Engelhardt KR, Holland S, Puck JM, Grimbacher B. An update on the hyper-IgE syndromes. Arthritis research \& therapy. 2012;14(6):1-10.

8. Aujla SJ, Chan YR, Zheng M, Fei M, Askew DJ, Pociask DA, et al. IL-22 mediates mucosal host defense against Gram-negative bacterial pneumonia. Nature medicine. 2008;14(3):275.

9. Avery DT, Ma CS, Bryant VL, Santner-Nanan B, Nanan R, Wong M, et al. STAT3 is required for IL-21-induced secretion of IgE from human naive B cells. Blood. 2008;112(5):1784-93.

10. Motedayyen H, Ghotloo S, Saffari M, Sattari M, Amid R. Evaluation of microRNA-146a and its targets in gingival tissues of patients with chronic periodontitis. Journal of periodontology. 2015;86(12):1380-5.

11. Ghotloo S, Motedayyen H, Amani D, Saffari M, Sattari M. Assessment of micro RNA-146a in generalized aggressive periodontitis and its association with disease severity. Journal of periodontal research. 2019;54(1):27-32.

12. Renner ED, Rylaarsdam S, Aňover-Sombke S, Rack AL, Reichenbach J, Carey JC, et al. Novel STAT3 mutations, reduced TH17 cell numbers, and variably defective STAT3 phosphorylation in Hyper-IgE syndrome. The Journal of allergy and clinical immunology. 2008;122(1):181.

13. Grimbacher B, Holland SM, Gallin JI, Greenberg F, Hill SC, Malech HL, et al. Hyper-IgE syndrome with recurrent infections-an autosomal dominant multisystem disorder. New England Journal of Medicine. 1999;340(9):692-702.

14. O'Connell AC, Puck JM, Grimbacher B, Facchetti F, Majorana A, Gallin JI, et al. Delayed eruption of permanent teeth in hyperimmunoglobulinemia E recurrent infection syndrome. Oral Surgery, Oral Medicine, Oral Pathology, Oral Radiology, and Endodontology. 2000;89(2):177-85.

15. Domingo D, Freeman A, Davis J, Puck J, Tianxia W, Holland S, et al. Novel intraoral phenotypes in hyperimmunoglobulin-E syndrome. Oral diseases. 2008;14(1):73-81.

16. Wjst M, Lichtner P, Meitinger T, Grimbacher B. STAT3 single-nucleotide polymorphisms and STAT3 mutations associated with hyper-IgE syndrome are not responsible for increased serum IgE serum levels in asthma families. European Journal of Human Genetics. 2009;17(3):352. 
17. Saeedfar K, Behmanesh M, Mortaz E, Masjedi MR. The Expression of STAT3 and STAT5A Genes in Severe Refractory Asthma. Tanaffos. 2017;16(1):1.

\section{Figure legend}

Figure. 1) Dental complications in a patient with AD-HIES.Permanent teeth erupt adjacent to primary teeth (double rows of teeth). 


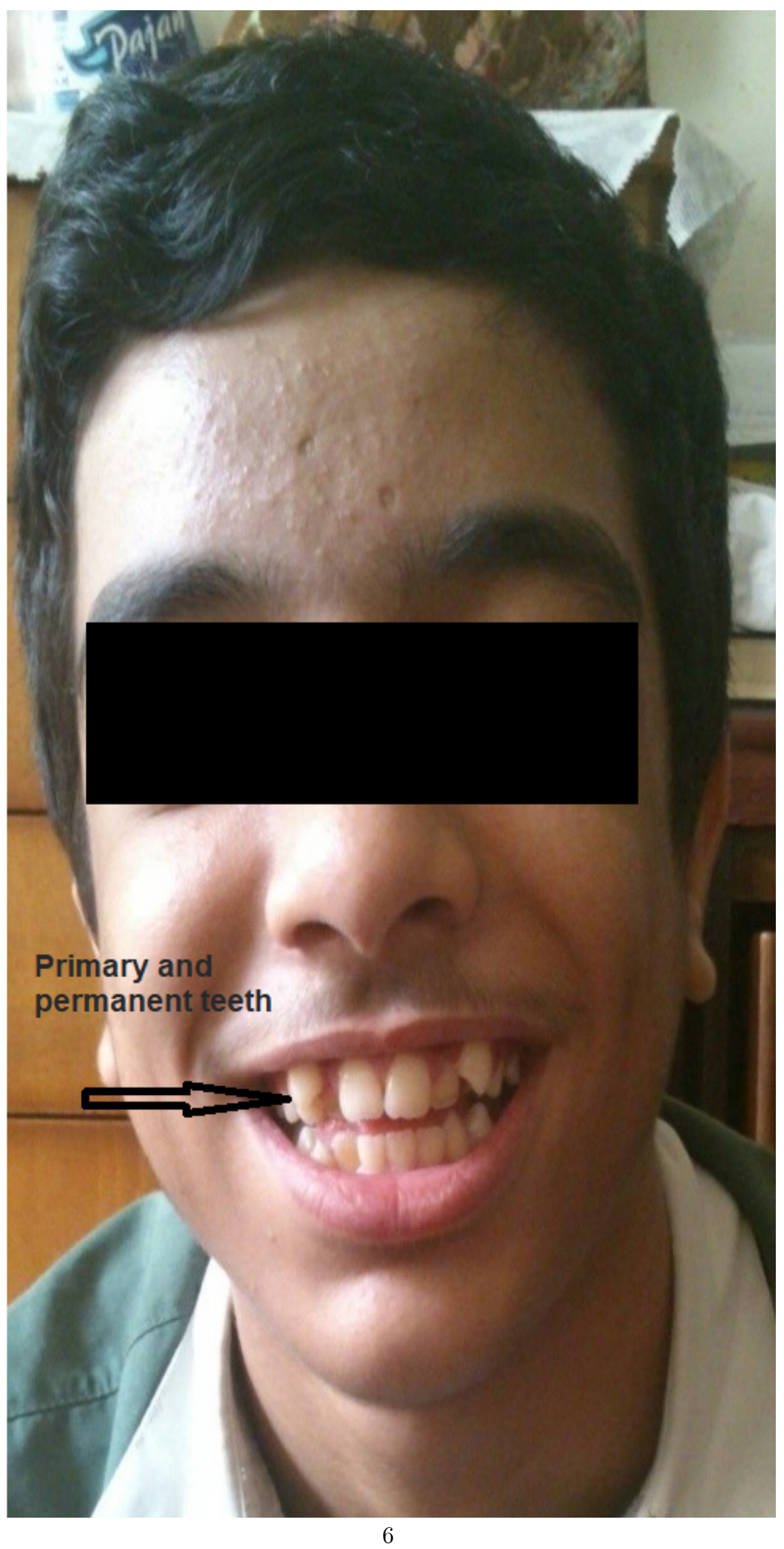

